\title{
A Healthier Beverage Choice is Based on a Subjective Assessment of Sweet Taste
}

\author{
Ester Reijnen ${ }^{1}$, Swen J. Kühne ${ }^{1} \&$ Reto Ritter ${ }^{1}$ \\ ${ }^{1}$ School of Applied Psychology, ZHAW Zurich University of Applied Sciences, Zürich, Switzerland \\ Correspondence: Ester Reijnen, School of Applied Psychology, ZHAW Zurich University of Applied Sciences, \\ Pfingstweidstrasse 96, Box 707, CH-8037-Zürich, Switzerland. E-mail: ester.reijnen@zhaw.ch
}

Received: January 23, 2021

Accepted: March 4, $2021 \quad$ Online Published: March 10, 2021

doi:10.5539/jfr.v10n2p47

URL: https://doi.org/10.5539/jfr.v10n2p47

\begin{abstract}
Despite promising interventions to lower people's daily sugar consumption, such as health- or taste-focused labels, the consumption of sugar-sweetened beverages (SSBs) continues to rise. To improve the effectiveness of existing labels, the way people process sugar amounts in grams $(\mathrm{g})$ as displayed on beverages seems to merit elucidation. For example, do people perceive the difference in the amount of sugar, and thus in the subjective sweet taste, between two beverages according to Weber's law? Additionally, is that perceived difference the cause of their beverage choice? In order to investigate these questions, participants in this online experiment first had to estimate the sugar difference between two beverages based on grams and then decide whether they would switch to a lower-sugar beverage. We found that participants' different estimates followed Weber's law. The choice of the lower-sugar beverage, however, depended on how large they personally perceived that difference. In other words, the choice was independent of the ratio. These results show that future labels, rather than indicating the total amount of sugar, should indicate whether the reduction, for example in the amount of sugar compared to another beverage, was perceived as significant by others.
\end{abstract}

Keywords: sugar-sweetened beverages, choice, ratio, individual perceived differences, taste

\section{Introduction}

Here's a dilemma for you: The $5 \mathrm{dl}$ "peach" iced tea ( $22.6 \mathrm{~g}$ sugar) or the $5 \mathrm{dl}$ "light peach" iced tea $(0.2 \mathrm{~g}$ sugar)? A choice not without consequences as more and more studies postulate a positive relationship between the consumption of sugary food items, especially sugar-sweetened beverages (SSBs), and obesity (see, for example, Luger et al., 2017). Although governments and other organizations have developed and implemented interventions to reduce the consumption of SSBs (e.g., from product reformulation to labels; see, for example, Di Monaco, Miele, Cabisidan, \& Cavella, 2018), its worldwide per capita consumption has continued to rise in many countries in recent years (Popkin \& Hawkes, 2016).

One of the most promising interventions so far in terms of acceptance (Hagmann, Siegrist, \& Hartmann, 2018, compare the acceptance of labels with other interventions such as taxation or reducing availability) as well as efficiency (meta-analysis of Cecchini \& Warin, 2016) is the use of so-called front-of-pack health labels such as the Traffic Light Label (TLL). The TLL allows for a quick classification of the food items' nutritional properties, either by color-coding the items' amount of sugar, salt, fat and saturates (i.e., multiple TLL; e.g., red = high), or its overall nutritional quality (i.e., single TLL). Although the TLL improves nutritional knowledge (see, for example, Roberto, et al., 2012), its influence on purchase intention or actual purchase of healthier food items, especially SSBs, is inconclusive. While, for example, Thorndike, Sonnenberg, Riis, Barraclough and Levy (2012) found that the TLL increased sales of healthy beverages and respectively reduced sales of unhealthy beverages, Findling, et al. (2018) found no effect of the TLL on participants' purchase intentions.

Turnwald and Crum (2019) attribute the lack of consistency in the effect of labels that lay their emphasis on the nutritional properties of a food item (such as the TLL or text-based labels that say "healthy choice") to the fact that those so called health-focused labels are not "tailored to people's preferences in the moment of food choice" (p. 8). Therefore, Turnwald and Crum (2019) suggested to concentrate on taste (i.e., here sweetness, modulated by the amount of sugar) rather than on nutritional properties. They showed that by using taste-focused labels (i.e., establishing an association of "healthy" with taste) instead of the health-focused labels mentioned before (e.g., the TLL), the choice of "healthy" food items (i.e., vegetables, salad, vegetable wraps, green beans) 
increased by $38 \%$. However, since the authors did not have a classical control group without a label, it is unclear where the performance of the labels, especially the health-focused label group, would have been in relation to a control group; the results of the control group would indicate the "uninfluenced" expected taste of the food items. Nonetheless, the results found by Turnwald and Crum (2019) indicate that the underlying mechanism for our food item choices is the expected taste (next to - though not the topic of this study - actual experienced taste). We thereby assume that expected taste is modulated differently depending on the label. Taste-focused labels (compared to a control group) should have neither a negative nor a positive influence on choice; this contrasts with health-focused labels. In some cases, these (health-focused) labels have a negative effect on choice, through a reduction in tastiness, as shown in Turnwald and Crum's (2019) study. In other cases, though, as shown by Wansink, Ittersum and Painter (2004), the reverse effect was observed, especially for desserts. Desserts were rated tastier when described as healthy. Our assumption that choice depends on taste perception, which is mainly modulated by nutritional information is supported, for example, by the study of Aggarwal, Rehm, Monsivais, and Drewnowski (2016). They showed that while about $77 \%$ of US adults thought that taste was a "very important" determinant for their dietary choices (compared to $59.9 \%$ for nutrition), it was nutrition that most strongly predicted their eating behavior. Thus, it is the health-focused labels that can manipulate our taste (while taste-focused labels just reflect our actual taste preference). Further investigation is needed to find out under which conditions health-focused labels can have a harmful or beneficial effect.

Pioneering this investigation requires that we know how consumers perceive the expected (sweet) taste of a beverage without label information, that is, only with the help of the numerical indication of the sugar quantity in " $g$ ". Moreover, a key feature which will be considered is the presence of a reference item. As stated by Dan Ariely in his noteworthy study from 2010 "we always look at things around us in relation to others" (p. 7). Ariely (2010) shows that items' attributes, such as how expensive a car is or how relaxing holidays are, are always perceived "in relation to each other" (never in isolation). Accordingly, this should also apply to the expected taste of a beverage. In our "iced tea" scenario presented at the beginning, this reference item could then be either one of the two iced teas (i.e., the "peach" iced tea with $22.6 \mathrm{~g}$ sugar or the "light peach" iced tea with only $0.2 \%$ sugar (Note a)). To the best of our knowledge, it has not yet been investigated whether the subjectively perceived difference in taste between two beverages - deduced from the stated amounts of sugar in grams - has an effect on the choice of the healthier beverage alternative, for example, the less sugary iced tea. To date, to the best of our knowledge, no empirical investigation has unveiled how the expected taste of two beverages that differ in how sugary and healthy they are, are perceived respective of how it influences choices, such as the chance to switch to a less sugary iced tea. For example, does the calculation of the perceived difference in the amount of sugar (and thereby the difference in taste or sweetness), follow ratio (according to Weber's law, see also Reijnen, Wolfe, and Krummenacher, 2013)? To illustrate it in an example: If ratio is relevant, the ability to discriminate between the expected taste of two sugar-sweetened drinks should decrease when the difference between two numbers becomes smaller (i.e., $1 \mathrm{~g}$ vs. $2 \mathrm{~g}$ compared to $1 \mathrm{~g}$ vs. $9 \mathrm{~g}$; distance effect) or when the two numbers become larger, though the distance is kept the same (i.e., $8 \mathrm{~g}$ versus $9 \mathrm{~g}$ versus $1 \mathrm{~g}$ versus $2 \mathrm{~g}$; size effect). Hence, the decision to switch to the "healthier" beverage should become more difficult.

Why do we focus on "ratio"? Studies of the actual experienced (compared to expected) sweetness in taste perception showed that Weber's Law plays a key role. For example, Chang and Chiou (2006) showed that the higher the initial sweetener level, the harder it was for consumers to tell the difference in sweetness. Interestingly, Chiou, Yeh and Chang (2009) showed that these experienced differences in sweetness perception can be influenced by labels. That is, they became smaller when a (health) label was present than when not, suggesting that consumers were more sensitive to sweetness under these conditions. Hence, another support for health-focused labels. Therefore, in this study we aim to answer the questions: do participants' differences in sugar perception follow ratio in accordance with Weber's law and consequentially, do these expected differences in taste become the drivers of their beverage choices?

\section{Method}

\subsection{Participants}

726 participants ranging in age from 18 to $52\left(M_{\text {age }}=25.1 ; S D_{\text {age }}=4.8 ; 62.5 \%\right.$ female $)$ from ZHAW Zurich University of Applied Sciences and the greater area of Zurich took part in this web-based study. Participants could take part in a lottery for an iPad and two cinema vouchers. Participants from the ZHAW School of Applied Psychology could instead receive course credit for their participation (4.5\% did). All participants gave informed consent. 


\subsection{Stimulus Material}

Two well-known carbonated beverages in Switzerland (Apfelsaft $=$ carbonated apple juice, Apfelschorle $=$ carbonated apple juice mixed with water, see Figure 1), which differ from each other essentially only in the amounts of sugar, were used as stimulus material and presented to the participants in picture form. In addition to the pictures, the amount of sugar for each beverage was displayed as a number in g / $100 \mathrm{ml}$ (e.g., Apfelsaft: 9.3 $\mathrm{g}$; Apfelschorle: $4.7 \mathrm{~g}$ ). These numbers were manipulated in such a way that six different $\mathrm{g}$ combinations of sugar amounts resulted. We chose the numbers in such a way that we could exclude alternative explanations as the cause of the participants' different ratings in perception, such as absolute difference (see Moyer \& Landauer, 1967 , for reaction time in comparing two numbers) in the amount of sugar between the two beverages or number of digits (before the semicolon; Reynvoet \& Brysbaert, 1999). Hence, as can be seen in Figure 1, in three conditions the absolute difference in sugar content was large (4.6 g), whereas in the other three it was small (2.6 $\mathrm{g}$; manipulation of the variable: absolute difference in the amount of sugar). Furthermore, in one third of the conditions the number of digits used in declaring the amount of sugar was one (i.e., single- vs. single-digit), in another third of the conditions it was mixed (i.e., double vs. single-digit) and in the last third of the conditions it was two (i.e., double- vs. double-digit; variable number of digits). The chosen numbers should also remain as close as possible to the actual amount of sugar (in grams) in the beverages (+/- $4.3 \mathrm{~g}$ max.). Based on the selected numbers or g-combinations, the following ratios (variable ratio; 0.51, 0.63, 0.70, 0.72, 0.77 and 0.80 ) resulted. Note that the participants' differences in perception of the amount of sugar contained in the two beverages were expected to follow these ratios (see next section: procedure).

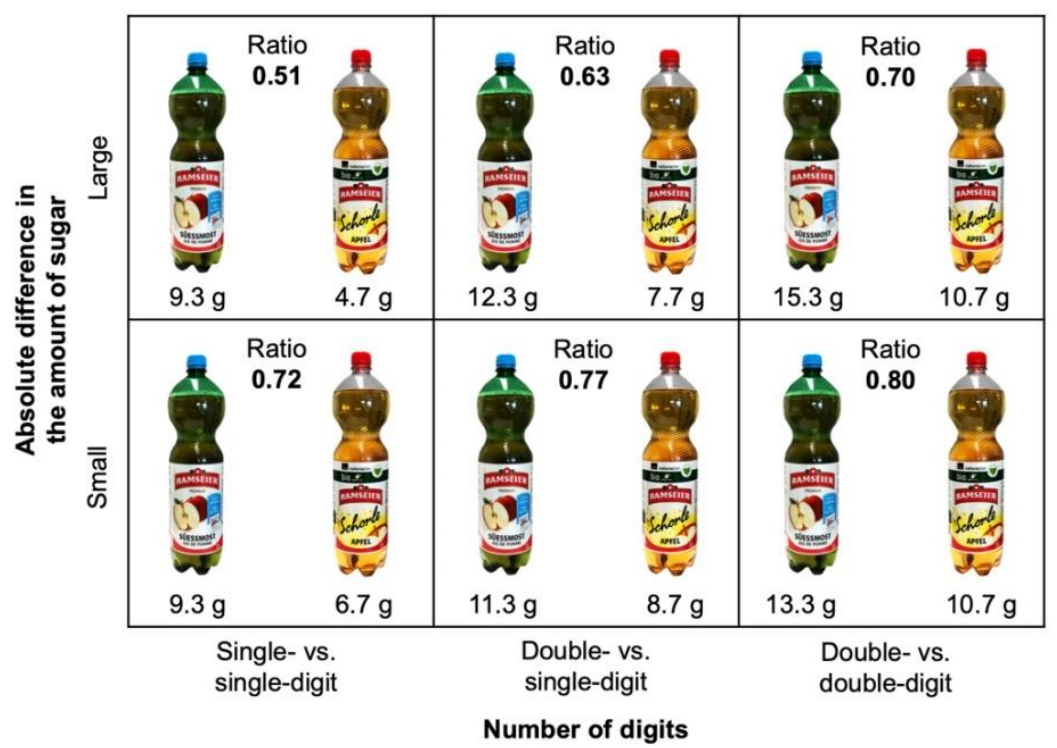

Figure 1. The six conditions

Note. The six conditions are separated by the factors number of digits and absolute difference in the amount of sugar. The appropriate ratios are displayed in bold.

\subsection{Procedure}

Participants were instructed to imagine that they were in a supermarket and had already put a specific beverage (Apfelsaft) in their shopping cart, when their attention was suddenly drawn to another beverage (Apfelschorle). The first beverage (Apfelsaft) thereby differed from the latter (Apfelschorle) due to its higher amount of sugar (for example, $13.3 \mathrm{~g}$ instead of $10.7 \mathrm{~g}$; see Figure 1)(Note 2). Note, that the respective beverages' amount of sugar was always displayed along with the beverages. After reading the initial description, participants had to state (variable: difference perception) how much more sugar the Apfelsaft (the beverage in their cart) had, relative to the Apfelschorle (the second beverage) on a 7 -point Likert scale $(1=$ very small; $7=$ very large). Thereafter, participants were asked whether they wanted to stick (binary yes / no choice) with the beverage already placed in their basket or if they wanted to switch to the less sugary beverage (variable: beverage choice). Participants were randomly assigned to one of the 6 ratio conditions $(0.51,0.63,0.70,0.72,0.77$ and 0.80$)$, thus controlling for all confounding variables, including the preference for one or the other beverage. Each participant, 
therefore, had to rate once and choose once. At the end, we assessed their demographic data (e.g., age, sex).

\subsection{Statistical Analysis}

All data analysis were carried out using R software (version 4.0.2). Binary linear regressions were performed by using the rms package (Harrell, 2021).

\section{Results}

\subsection{Participants Excluded}

Participants who needed more than 30 or less than 1.5 minutes to complete the whole experiment and/or took more than 300 or less than 5 seconds (altogether $3.1 \%$ ) to complete the difference rating and choice task were excluded from the analysis.

\subsection{Difference Perception}

The calculated linear regression showed that participant differences in perceptions linearly followed ratio, $F(1$, $724)=42.08, p<.001, R_{a d j}^{2}=0.05$ (see Figure 2); that is, the smaller the ratio the smaller the perceived difference in the amount of sugar.

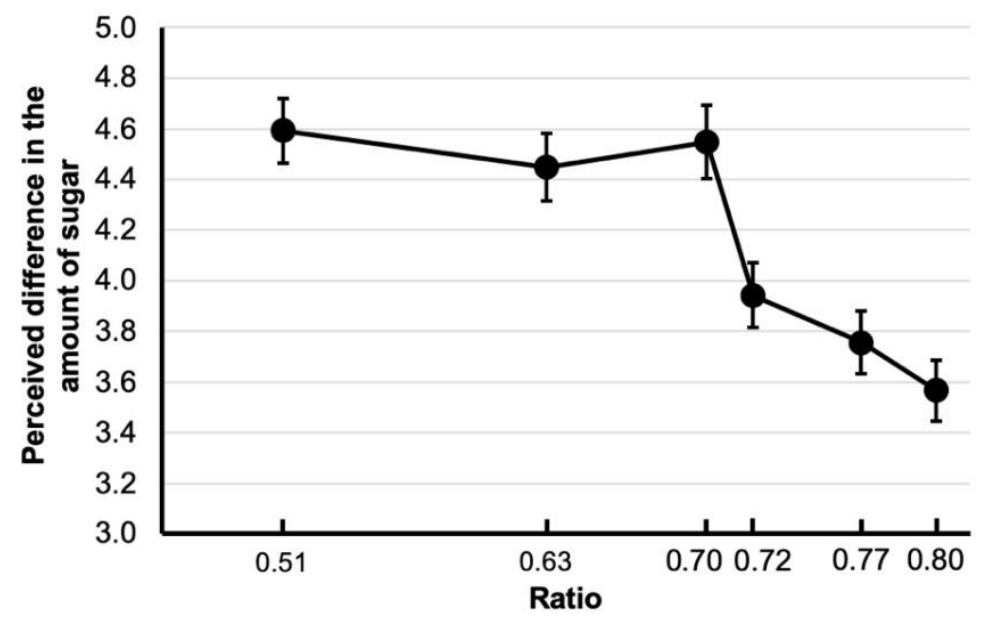

Figure 2. Perceived difference in sugar content per ratio

Note. Bars indicate standard errors (for statistical details see section "3.2. Difference Perception”).

\subsection{Beverage Choice}

Whether ratio is a predictor for choice was tested by using a binary logistic regression. Surprisingly, ratio does not seem to be predictive of participants' beverage choices, that is, whether they kept the Apfelsaft or switched to the Apfelschorle; $\left.\chi^{2}(1, N=726)=0.64, p=.42\right)($ Note 3$)$. Moreover, in all ratio conditions the percentage of choosing Apfelschorle is at chance (i.e., $50 \%$; all $\chi^{2}$ s $<2.23$, all $p$ 's $>.13$; except for the ratio condition $=0.51$, in which participants switched to Apfelschorle in about $60 \%$ of the cases; $\left.\chi^{2}(1, N=125)=4.61, p<.05\right)$. This fact is illustrated in Figure 3, where in each ratio condition (except the 0.51 condition) there is a similar number of circles (Apfelsaft choices) and triangles (Apfelschorle choices). 


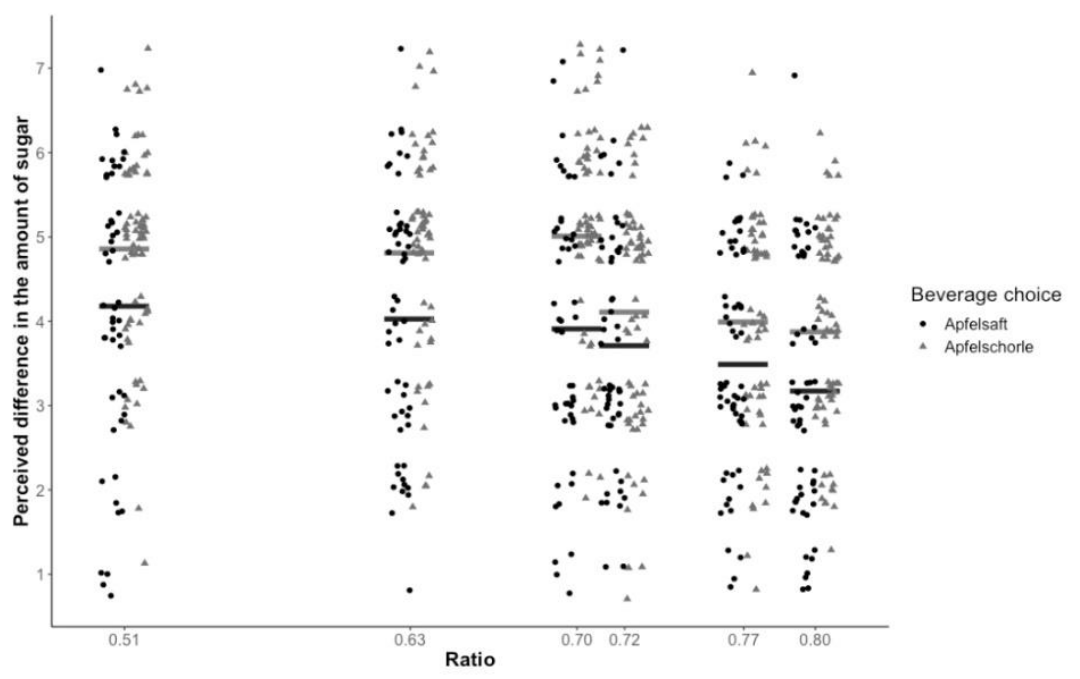

Figure 3. Scatterplots for the perceived differences in the amount of sugar per participant

Note. Each circle (Apfelsaft) or triangle (Apfelschorle) represents a participant and the specific beverage he/she has chosen. Small jittering was added to each data point (circle / triangle) for better readability of the figure (so circle / triangles don't overlap). The vertical lines per ratio represent the group means in the perceived differences in the amount of sugar for choosing Apfelsaft or Apfelschorle. Ratio is not a significant predictor for choice.

However, Figure 3 also shows that the larger the perceived difference the more participants switch to the Apfelschorle. This observation was confirmed by the logistic regression: $\chi^{2}(1, N=726)=39.84, p<.001, R^{2}=$ 0.08 ; indicating that the perceived difference in the amount of sugar is, independent of the condition at hand, a valid predictor for choice behavior (see Figure 4 for illustrative reasons). The stepwise inclusion of the factor ratio and its interaction with the perceived difference could not add any value to the model (both likelihood ratio tests: $\chi^{2}$ 's $<0.56, p$ 's $>.45$ ) and they were thereby omitted in the model.

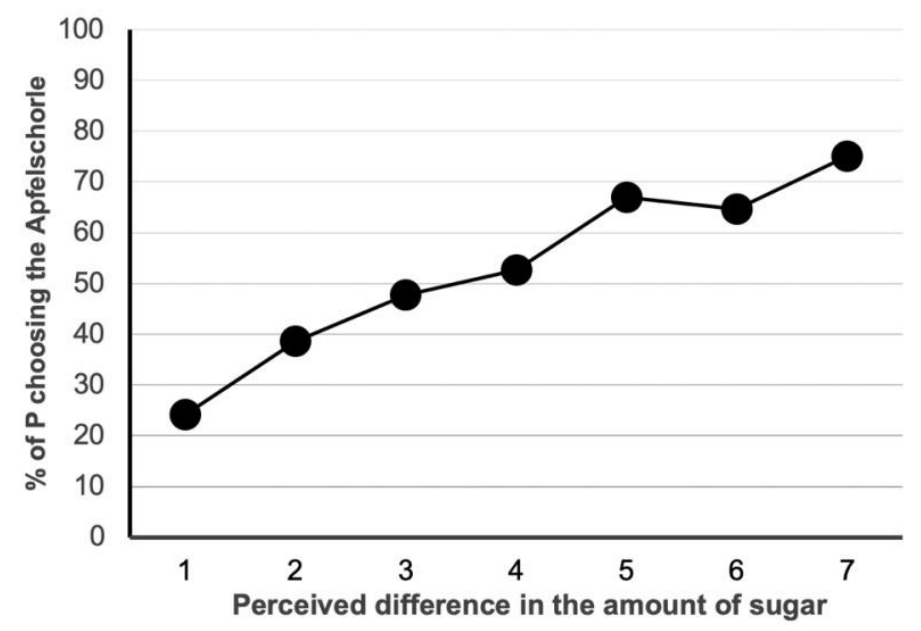

Figure 4. Participants choice depending on the perceived difference

Note. Points indicate the percentage of participants who switched to Apfelschorle for each value on the scale of the perceived difference in the amount of sugar $(1=$ very small; $7=$ very large difference). Thereby the percentage of participants who switched to Apfelschorle increases linearly with the perceived difference in the amount of sugar.

\section{Conclusion and Discussion}

Our results demonstrate that ratio nicely predicts the average perceived sugar difference (or average difference in 
expected taste) between two beverages. However, ratio was not predictive of choice behavior, but rather of how participants perceived the sugar difference subjectively. In other words, regardless of the given ratio (e.g., small: 0.51 or large: 0.80 ), participants only switched to the less sugary beverage if the (objective) difference between the two beverages was subjectively perceived as large (rated with, for example, a 7 on the Likert scale). Hence, though participants were well equipped to calculate differences, these numerical measures were useless when it came to their choices. It only mattered how the participants perceived that sugar difference or difference in expected taste subjectively.

Considering these insights, how can we begin to influence these individually perceived differences in sweetness taste? One option could be to add a slogan such as "others perceived the sugar reduction as meaningful" (see Cialdini et. al, 2006, about the persuasive impact of social norms). This social norm may turn a difference, perceived as "small" by an individual, into a large difference. This should not lead to a reduction in the expected taste, if the effect is like that of the desserts mentioned in the study of Wansink, Ittersum and Painter (2004) in our introduction.

How can we now also use these insights to improve the effectiveness of health-related labels? As already mentioned in the introduction, taste information is part of every food item choice. However, health information, although well camouflaged, is also part of it; note that we are not talking about health information provided by health-related labels. How does this camouflage come about? In this respect, let us look at the study by Sullivan, Hutcherson, Harris and Rangel (2015), in which participants had to make a binary choice between two randomly selected food items. Pairs could be any combination of taste and health. By using a new type of mouse tracking, they were able to investigate how the two attributes (taste and health) are processed over time and integrated into the choice process. They found that since taste information is processed about 195 milliseconds before health information, the latter (i.e., health) information has less weight/influence regarding the choice of food items. Transferring the findings of Sullivan et al. (2015) to the TLL, the TLLs color coding (color is processed early in the perceptual system) most likely modulates the taste information, leading to the effects described in the introduction. However, this is most probably not the intended idea of health-related labels.

How can the TLL now influence health-related information? This can be accomplished by designing the choice situation in such a way that health-focused labels (and thus health information) are processed before the processing of taste information even begins. For example, a supermarket could put up signs such as "healthy food corner" and arrange the product items accordingly. This could lead to consumers processing "health" information first.

However, the TLL has 2 more weaknesses. The first is that sugar differences (here the objective, not even the subjective ones) are sometimes, or sometimes not, adequately indicated by the color-coding. Let's consider, for example, the choice between a veePRO bar $(15 \mathrm{~g} / 100 \mathrm{~g})$ and a Farmer natural bar $(3 \mathrm{~g} / 100 \mathrm{~g})$. The $12 \mathrm{~g}$ sugar difference is adequately represented here by the corresponding TLL colors(Note 4): While the veePRO bar has an orange sugar label, the farmer natural bar has a green one. Note, the TLL colors should inform consumers quickly about the health status of the food item (e.g., green = healthy). Now let's consider the choice between a Snickers $(47 \mathrm{~g} / 100 \mathrm{~g})$ and a Clif Bar $(28 \mathrm{~g} / 100 \mathrm{~g})$. Here the even greater difference in the sugar values is not adequately represented in the TLL colors. Both bars have a red sugar label.

Let us now look at the second weakness, with the help of an example. French fries are classified as healthier (orange in fat and saturates) than nuts (red in fat and saturates; the other two nutrients are the same as in French fries). However, considering the quantity of these food items that consumers eat (the portion sizes are $28 \mathrm{~g}$ for nuts and $120 \mathrm{~g}$ for French fries), the assumption that French fries are healthier is no longer valid.

Given the weaknesses of TLLs, what other interventions, which can be summarized under the so-called "nudge" approach (for an overview in the food domain see Bucher et al., 2016), can be used alongside labels (or the slogan) to combat the world-wide obesity crisis successfully? A nudge is defined as "any aspect of the choice architecture that alters people's behavior in a predictable way without forbidding any options or significantly changing their economic incentives" (Thaler \& Sunstein, 2009, p. 6). Banning SSB's or making them much more expensive is therefore not a nudge.

A promising nudge is the relative positioning of objects, here of food items. For example, Reijnen, Kühne, von Gugelberg and Crameri (2019) have shown that if you like the cooking style (e.g., Italian) of the restaurant in which you dine out, you prefer to select the menu placed in the middle. Hence, one might consider placing the sugar-free beverages in preferred positions in the supermarket. In addition, sugar-free drinks could act as an anchor (another nudge; see Tversky \& Kahneman, 1974) which should also encourage consumers to choose less sugary beverages. 


\subsection{Determinants of Participants Subjective Difference Perceptions}

As shown, a reduction in sugar in SSBs is accompanied by a reduction in taste liking. In consumer perception studies this is thought to be mediated by a change in participants' perception of the sensory properties of SSBs (e.g., color, sweetness, thickness - captured via a check-all-that-apply CATA question). Considering Civille and Oftedal's (2012) statement that "Products' sensory properties ... must be tailored to ultimately appeal to the "consumer": no matter how healthy and nutritious a food is, if it does not appeal to its intended end user, it is unlikely to succeed in today's marketplace." (p. 598) assessing these properties as well as their effect, is critical to developing SSBs that appeal to consumers. It could therefore be seen as a study limitation that these properties were not assessed.

But what do we know so far from consumer perception studies about these properties and their effect? First, if the sugar reduction is below the "just perceptible difference" (see Fechner, 1860) no differences in the perception of sensory properties of SSBs and taste liking are evident (see Lima, Ares, \& Deliza, 2017). Second, Oliveira, Ares, and Deliza (2018) also found no differential effects of sugar reduction in SSBs (20\% vs. 40\%) on sensory perception and (in contrast to our study) on taste liking, as far as expected taste liking was concerned. However, once it came to experienced taste liking, identical sugar reductions in SSBs resulted in a decreased frequency of use of the terms "sweet" and "very sweet" respectively an increased frequency of use of the terms "barely sweet" and "very sour" (uncorrected p-values) and a decrease in taste liking. So, it seems to matter how taste perception is measured. Most important, however, are the findings that consumers respond rather heterogeneously (as also shown in our study) to sugar reduction. In this sense, Oliveira et al. (2018) and Lima et al. (2017) showed that consumers can be classified into either a group that dislikes sugar-reduced SSBs or one who actually prefers sugar-reduced SSBs. Rather than focusing on the sensory perception of SSBs (and its effect), it is more appropriate to address the question of what factors lead to the distinction between these two groups. The only one found so far by Lima et al. (2017) is "education"; the group that preferred sugar-reduced SSBs had a higher proportion of adults with a high school or university degree.

In summary, consumer perception studies cannot explain why we found differences in perceptions. Our hypothesis is that they are driven by participants drinking habits (i.e., did they choose, for example, the Apfelsaft because they always choose it and to reduce cognitive dissonance rated the difference in the amount of sugar as small). Therefore, further experiments are necessary, in a broader sense too, to support our results found so far.

To sum up our results, the actual difference in the amount of sugar is not the key for consumers switching to a healthier beverage, but their perception of this difference as large is. The question now is, how one can present actual large differences in the amount of sugar in a way that consumers also perceive them as large? This study just closed one of the many research gaps.

\section{Author Contributions}

All authors listed have made an equal substantial, direct and intellectual contribution to the work, and approved it for publication.

\section{References}

Aggarwal, A., Rehm, C. D., Monsivais, P., \& Drewnowski, A. (2016). Importance of taste, nutrition, cost and convenience in relation to diet quality: Evidence of nutrition resilience among US adults using National Health and Nutrition Examination Survey (NHANES) 2007-2010. Preventive Medicine, 90, 184-192. https://doi.org/10.1016/j.ypmed.2016.06.030

Ariely, D. (2010). Predictably irrational: The Hidden Forces That Shape Our Decisions. New York, NY: Harper. https://doi.org/10.1037/e653632011-003

Bucher, T., Collins, C., Rollo, M. E., McCaffrey, T. A., De Vlieger, N., Van der Bend, D., ... Perez-Cueto, F. J. (2016). Nudging consumers towards healthier choices: a systematic review of positional influences on food choice. British Journal of Nutrition, 115, 2252-2263. https://doi.org/10.1017/S0007114516001653

Cecchini, M., \& Warin, L. (2016). Impact of food labelling systems on food choices and eating behaviours: a systematic review and meta-analysis of randomized studies. Obesity Reviews, 17(3), 201-210. https://doi.org/10.1111/obr.12364

Chang, M. H., \& Chiou, W. B. (2006). Differential threshold and psychophysical power function of sweetness sensation: Applied psychophysics and prospect theory on formulating baking products. Journal of Sensory Studies, 21, 534-551. https://doi.org/10.1111/j.1745-459X.2006.00078.x

Chiou, W. B., Yeh, L. T., \& Chang, M. H. (2009). Effects of health-related claims on the differential threshold of 
consumers' sweetness sensation. Journal of Sensory Studies, 24, 621-633. https://doi.org/10.1111/j.1745-459X.2009.00230.x

Cialdini, R. B., Demaine, L. J., Sagarin, B. J., Barrett, D. W., Rhoads, K., \& Winter, P. L. (2006). Managing social norms for persuasive impact. Social Influence, 1, 3-15. https://doi.org/10.1080/15534510500181459

Civille, G. V., \& Oftedal, K. N. (2012). Sensory evaluation techniques-Make "good for you" taste "good". Physiology \& Behavior, 107(4), 598-605. https://doi.org/10.1016/j.physbeh.2012.04.015

Di Monaco, R., Miele, N. A., Cabisidan, E. K., \& Cavella, S. (2018). Strategies to reduce sugars in food. Current Opinion in Food Science, 19, 92-97. https://doi.org/10.1016/j.cofs.2018.03.008

Fechner, G. T. (1860). Elemente der psychophysik (Vol. 2). Breitkopf u. Härtel.

Findling, M. T. G., Werth, P. M., Musicus, A. A., Bragg, M. A., Graham, D. J., Elbel, B., \& Roberto, C. A. (2018). Comparing five front-of-pack nutrition labels' influence on consumers' perceptions and purchase intentions. Preventive Medicine, 106, 114-121. https://doi.org/10.1016/j.ypmed.2017.10.022

Food Standards Agency. (2016). Guide to creating a front of pack (FoP) nutrition label for pre-packed products sold through retail outlets. Retrieved from https://assets.publishing.service.gov.uk/government/uploads/system/uploads/attachment_data/file/566251/F oP_Nutrition_labelling_UK_guidance.pdf

Hagmann, D., Siegrist, M., \& Hartmann, C. (2018). Taxes, labels, or nudges? Public acceptance of various interventions designed to reduce sugar intake. Food Policy, 79, 156-165. https://doi.org/10.1016/j.foodpol.2018.06.008

Harrell, F., Jr. (2021). Regression modeling strategies (Version 6.1-1). Retrieved from https://hbiostat.org/R/rms/

Lima, M., Ares, G., \& Deliza, R. (2018). Children and adults' sensory and hedonic perception of added sugar reduction in grape nectar. Journal of Sensory Studies, 33(2), e12317. https://doi.org/10.1111/joss.12317

Luger, M., Lafontan, M., Bes-Rastrollo, M., Winzer, E., Yumuk, V., \& Farpour-Lambert, N. (2017). Sugar-sweetened beverages and weight gain in children and adults: A systematic review from 2013 to 2015 and a comparison with previous studies. Obesity Facts, 10, 674-693. https://doi.org/10.1159/000484566

Moyer, R. S., \& Landauer, T. K. (1967). Time required for judgements of numerical inequality. Nature, 215, 1519-1520. https://doi.org/10.1038/2151519a0

Oliveira, D., Ares, G., \& Deliza, R. (2018). The effect of health/hedonic claims on consumer hedonic and sensory perception of sugar reduction: Case study with orange/passionfruit nectars. Food Research International, 108, 111-118. https://doi.org/10.1016/j.foodres.2018.03.003

Popkin, B. M., \& Hawkes, C. (2016). Sweetening of the global diet, particularly beverages: patterns, trends, and policy responses for diabetes prevention. The Lancet Diabetes \& Endocrinology, 4, 174-186. https://doi.org/10.1016/S2213-8587(15)00419-2

Reijnen, E., Kühne, S. J., von Gugelberg, H. M., \& Crameri, A. (2019). Nudged to a menu position: the role of "I'm loving it"!. Journal of Consumer Policy, 42, 441-453. https://doi.org/10.1007/s10603-019-09413-4

Reijnen, E., Wolfe, J. M., \& Krummenacher, J. (2013). Coarse guidance by numerosity in visual search. Attention, Perception, \& Psychophysics, 75, 16-28. https://doi.org/10.3758/s13414-012-0379-8

Reynvoet, B., \& Brysbaert, M. (1999). Single-digit and two-digit Arabic numerals address the same semantic number line. Cognition, 72, 191-201. https://doi.org/10.1016/S0010-0277(99)00048-7

Roberto, C. A., Bragg, M. A., Schwartz, M. B., Seamans, M. J., Musicus, A., Novak, N., \& Brownell, K. D. (2012). Facts up front versus traffic light food labels: A randomized controlled trial. American Journal of Preventive Medicine, 43, 134-141. https://doi.org/10.1016/j.amepre.2012.04.022

Sullivan, N., Hutcherson, C., Harris, A., \& Rangel, A. (2015). Dietary self-control is related to the speed with which attributes of healthfulness and tastiness are processed. Psychological Science, 26, 122-134. https://doi.org/10.1177/0956797614559543

Thaler, R. H., \& Sunstein, C. R. (2009). Nudge: Improving decisions about health, wealth, and happiness. London: Penguin.

Thorndike, A. N., Sonnenberg, L., Riis, J., Barraclough, S., \& Levy, D. E. (2012). A 2-phase labeling and choice architecture intervention to improve healthy food and beverage choices. American Journal of Public Health, 102, 527-533. https://doi.org/10.2105/AJPH.2011.300391 
Turnwald, B. P., \& Crum, A. J. (2019). Smart food policy for healthy food labeling: Leading with taste, not healthiness, to shift consumption and enjoyment of healthy foods. Preventive Medicine, 119, 7-13. https://doi.org/10.1016/j.ypmed.2018.11.021

Tversky, A., \& Kahneman, D. (1974). Judgment under uncertainty: Heuristics and biases. Science, 185, 1124-1131. https://doi.org/10.1037/e301492005-001

Wansink, B., van Ittersum, K., \& Painter, J. E. (2004). How diet and health labels influence taste and satiation. Journal of Food Science, 69, 340-346. https://doi.org/10.1111/j.1365-2621.2004.tb09946.x

\section{Notes}

Note 1. It could also be an internal taste reference.

Note 2. Since we wanted to investigate the circumstances when participants switched to the healthier beverage alternative and not vice versa, the more sugary beverage was always placed in the shopping cart first.

Note 3. Also, the factor's number of digits and difference in sugar content are not predictive regarding choice (both main effects and their interaction: $\chi^{2}$ 's $<1.34, p$ 's $>.51$ ).

Note 4. TLL colors (e.g., for sugar, salt, etc.) are defined by the Food Standards Agency (2016).

\section{Copyrights}

Copyright for this article is retained by the author(s), with first publication rights granted to the journal.

This is an open-access article distributed under the terms and conditions of the Creative Commons Attribution license (http://creativecommons.org/licenses/by/4.0/). 